\title{
Emerging Tunnel FET and Spintronics based Hardware Secure Circuit Design with Ultra-low Energy Consumption
}

\section{Aditya Japa ( $\sim$ aditya@iiitnr.edu.in )}

Dr Shyama Prasad Mukherjee International Institute of Information Technology https://orcid.org/00000003-2408-5400

\section{Subhendu K. Sahoo}

BITS Pilani: Birla Institute of Technology and Science

\section{Ramesh Vaddi}

SRM University AP - Amaravati

\section{Manoj Kumar Majumder}

Dr Shyama Prasad Mukherjee International Institute of Information Technology

\section{Research Article}

Keywords: Tunnel FET (TFET), Differential power analysis (DPA), Sense amplifier based logic (SABL), Spin transfer torque magnetic tunnel junction (STT-MTJ), Logic-in-memory (LiM), Logic encryption/locking

Posted Date: May 28th, 2021

DOl: https://doi.org/10.21203/rs.3.rs-262353/v1

License: (c) (i) This work is licensed under a Creative Commons Attribution 4.0 International License. Read Full License 


\title{
Emerging Tunnel FET and Spintronics based Hardware Secure Circuit Design with Ultra-low Energy Consumption
}

\author{
Aditya Japa $・$ Subhendu K. Sahoo $・$ Ramesh Vaddi $・$ Manoj Kumar Majumder
}

\begin{abstract}
Present CMOS technology with scaled channel lengths exhibited higher energy consumption in designing secure electronic circuits against hardware vulnerabilities and breaches. Specifically, CMOS sense amplifier based secure differential power analysis (DPA) countermeasures at scaled channel lengths show large energy consumption with increased vulnerability. Additionally, spin transfer torque magnetic tunnel junction (STT-MTJ) and CMOS based logic-in-memory (LiM) cells demonstrate high energy consumption due to the large write current requirement of STT-MTJ and poor MOS device performance at scaled channel lengths. This paper for the first time leverages emerging tunnel FET (TFET) steep-slope device characteristics and compatible non-volatile STT-MTJ devices for enhanced hardware security with ultra-low energy consumption at lower supply voltages. TFET based sense amplifier based logic (SABL) gates have been proposed that achieve $3 \times$ lower energy consumption compared to Si FinFET SABL designs. Further, utilizing TFET SABL gates, TFET Pride S-box is designed that exhibits higher DPA resilience with $3.2 \times$ lower energy consumption compared to FinFET designs. With resulted lower static power consumption, TFET SABL based crypto systems can show lower vulnerability to static power side-channel attacks. Besides, proposed STT-MTJ and TFET LiM gates achieves $4 \times$ lower energy consumption compared to STT-MTJ and FinFET designs. Moreover, these gates have been explored in logic encryption/locking technique that shows $3.1 \times$ lower energy consumption compared to STT-MTJ and FinFET based design.
\end{abstract}

Keywords Tunnel FET (TFET) - Differential power analysis (DPA) - Sense amplifier based logic (SABL) - Spin transfer torque magnetic tunnel junction $(\mathrm{STT}-\mathrm{MTJ}) \cdot \operatorname{Logic}$-in-memory $(\mathrm{LiM}) \cdot \operatorname{Logic}$ encryption/locking

\section{Introduction}

Aditya Japa ${ }^{1}$

$\underline{\text { aditya@iiitnr.edu.in }}$

Subhendu K. Sahoo ${ }^{2}$

sahooemailid@gmail.com

Ramesh Vaddi ${ }^{3}$

rameshvaddi2k9@gmail.com

Manoj Kumar Majumder ${ }^{1}$

manojk@iiitnr.edu.in

${ }^{1}$ Department of Electronics and Communication Engineering, DSPM International Institute of Information Technology, Naya Raipur 493661, India.

${ }^{2}$ Department of Electrical and Electronics Engineering, Birla Institute of Technology and Science, Hyderabad 500078, India.

${ }^{3}$ Department of Electronics and Communication, School of Engineering and Applied Sciences, SRM University, Amaravati, Guntur 522502, Andhra Pradesh, India.

Offshore manufacturing of integrated circuits (ICs) show increased vulnerability to hardware security attacks in the modern internet of things (IoT) era [1-2]. In support of this, the national institute of standards and technology has observed an exponential growth in hardware vulnerabilities in the last few years [3]. Moreover, the hardware security aided techniques or systems market is growing rapidly to avoid the counterfeiting of ICs [4]. There exist diversified hardware security attacks in the literature including hardware reverse engineering, counterfeiting of IC, and side-channel analysis (SCA). Among different hardware security attacks available, the SCA is effective in recovering the hidden secret information [5]. SCA utilizes the side-channel signals like power consumption, electromagnetic and photonic signals to reveal encryption key of the cryptographic circuits/systems [6]. Differential power analysis (DPA) process the power consumption information of encryption engine and require comparatively less effort in recovering secret key information [7]. Additionally, static CMOS logic style shows higher vulnerability to DPA attacks due to the data dependent power consumption profile [8-9]. To avoid this, several researchers explored novel logic styles that show higher DPA resilience [10]. Sense amplifier based logic 
(SABL), FinFET based secure adiabatic logic (FinSAL), randomized multi-topology logic (RMTL), homogeneous dual-rail logic (HDRL), secure positive feedback adiabatic logic, and differential symmetric pull-down network gates have been demonstrated to mitigate the data dependency on device power consumption [11-16]. Among the novel logic designs, SABL logic style is proved to be secure and show several challenges including higher power and energy consumption overheads [11]. Moreover, at sub$50 \mathrm{~nm}$ channel lengths, CMOS SABL exhibited higher static power consumption that revealed the confidential information against static power SCA [17]. To avoid this, emerging hyperFET devices have been leveraged for DPA resilient SABL logic. However, this technique has exhibited relatively $3 \times$ higher energy consumption compared to CMOS [18]. Thus, CMOS SABL logic shown unavoidable disadvantages that need to be addressed for DPA resilient crypto graphic circuits with ultra-low energy consumption.

On the other hand, spin transfer torque based magnetic tunnel junction (STT-MTJ) has attracted wide attention due to their lower static power, non-volatility and higher compatibility with CMOS technology [19-20]. Moreover, integrating STT-MTJ and CMOS provided flexibility to build logic-in-memory (LiM) architectures for high speed data transfer [21]. Several researchers demonstrated logic gates and arithmetic circuits by exploring STT-MTJ based LiM cells [22-23]. However, STT-MTJ require relatively large write current to change the state that demands to maintain higher supply voltages [24]. Besides, CMOS technology scaling lead to serious problems including lower ON current, short channel effects, high power density, and unreliability [25]. Consequently, the STTMTJ based LiM circuits exhibited large energy consumption due to the poor performance of MOS devices at scaled channel lengths (or supply voltages). In support of this, recent STT-MTJ LiM cells based crypto circuits exhibited increased energy consumption [26].

In the recent past, emerging tunnel FET (TFET) with its band-to-band tunnelling mechanism exhibited steep-slope characteristics (lower subthreshold swing) and higher ON to OFF current ratio ( $\left.\mathrm{I}_{\mathrm{ON}} / \mathrm{I}_{\mathrm{OFF}}\right)$ [27-29]. As a result, TFET based digital, analog and mixed signal circuits/systems achieved higher energy efficiency at lower supply voltages [30-31]. Apart from the low energy consumption and higher speed benefits, TFET devices with the special characteristics have been leveraged to enhance hardware security [32]. TFET based current mode logic and adiabatic logic have been proposed for differential power analysis resistant crypto systems [33-34]. TFET based compact polymorphic logic gates were designed by exploring the onset of tunnelling for both positive and negative gate bias [35]. The $\mathrm{p}-\mathrm{i}-\mathrm{n}$ forward current of TFET is explored as favourable feature to design true random number generator and low area overhead DPA countermeasure [36-37]. Thus, at scaled supply voltages, emerging TFET device has utilized to enhance hardware security of electronic systems [38]. Therefore, this work leverages TFET device characteristics for hardware security techniques that exhibit lower energy consumption. The SABL logic gates have been designed by exploring TFET device to reduce the energy consumption. The proposed TFET SABL gates are applied to design PRIDE S-box. Further, DPA attack is performed on proposed TFET SABL based S-box and also the performance is benchmarked with the base line Si FinFET designs. Additionally, STT-MTJ and TFET based LiM gates have been proposed to reduce the energy consumption. These designs have also benchmarked with STT-MTJ and FinFET based LiM gates. Moreover, the proposed LiM gates have been utilized in logic encryption/locking to obtain enhanced hardware security with ultra-low energy consumption.

The rest of the paper is organized as follows: Section 1 demonstrates the state-of-the art and the motivation of the proposed work. Section 2 presents the device characteristics of TFET and STT-MTJ that are explored for circuit design. Section 3 demonstrates TFET SABL based logic gates and Sbox design for DPA analysis. Further, these TFET SABL designs have been benchmarked with FinFET designs. Section 4 presents STT-MTJ and TFET based LiM gates and logic encryption/locking technique. Moreover, the performance of the STT-MTJ and TFET based logic encryption circuit is compared with STT-MTJ and FinFET designs. The fabrication compatibility of TFET and STT-MTJ technologies is also discussed in this section. Finally, conclusions are drawn in Section 5.

\section{Device Characteristics}

This section presents device characteristics, Verilog-A models of TFET and STT-MTJ that are required for circuit design. Further, the motivation to introduce TFET into SABL logic and STT-MTJ LiM circuits have also been discussed.

\subsection{TFET Technology}

TFET device works based on band-to-band tunnelling mechanism and exhibits high ON to OFF current ratio. Several TFET devices experimentally demonstrated lower subthreshold swing i.e., below $60 \mathrm{mV} / \mathrm{dec}$ [28-29]. This work explores LUT based 20nm InAs TFET Verilog-A models for circuit design [39]. The TFET based circuits have been benchmarked with equivalent LUT based 20nm Si FinFET designs [39]. The device architectures of $n$-channel TFET ( $N$ TFET) and n-channel Si-FinFET (N-FinFET) are as shown in Fig. 1. Various device parameters considered in this model are shown in Table 1. TFET and FinFET symbols were created by calling Verilog-A models into the industry standard Cadence tool. Later, circuits have been designed using the device symbols created. Figure 2 shows the $I_{D^{-}} V_{G S}$ characteristics of both N-TFET andN-FinFET by varying gate-to-source voltage $\left(V_{G S}\right)$ from 0 to $0.4 \mathrm{~V}$. Initially, when N-TFET switches OFF, it shows $2 \times$ lower OFF current compared to N-FinFET. With 


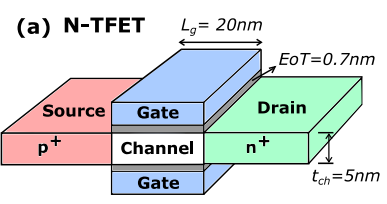

(b) N-FinFET

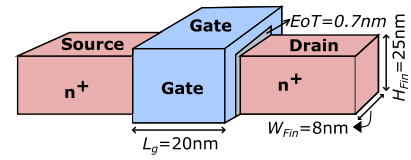

Fig. 1 Device architectures of (a) N-TFET (b) N-FinFET

sufficiently positive $V_{G S}$ voltage, N-TFET switches ON and current through N-TFET increases abruptly due to the band-to-band tunnelling mechanism. From Fig. 2(a), it can be observed that N-TFET shows $3.4 \times$ and $1.23 \times$ higher $\mathrm{ON}$ current at $V_{G S}$ of $0.3 \mathrm{~V}$ and $0.4 \mathrm{~V}$ respectively. Further, NTFET exhibits lower subthreshold swing $(30 \mathrm{mV} / \mathrm{dec})$ compared to N-FinFET device as shown in Fig. 2(b). From this, it can be concluded that, NTFET exhibits abrupt transition from OFF-state to ON-state (steep slope characteristics). TFET based circuits also achieve lower energy consumption due to high ON to OFF current ratio.

Recently, it has been revealed that SABL circuits become secure against DPA with the abrupt transitions from pre-charge to evaluation phase [18]. This behaviour requires abrupt transitions in the device characteristics that are used for circuit design. However, introduction of emerging steep-slope hyperFET devices in SABL based crypto system exhibited $3 \times$ higher energy consumption compared to CMOS based designs [18]. To reduce this energy consumption, this paper introduces steep-slope TFET devices in SABL circuits that can achieve ultra-low energy consumption with enhanced security assurance.

\subsection{STT-MTJ Device Characteristics}

Magnetic tunnel junction (MTJ) is a nano-stacked structure that consists of two ferromagnetic (FM) layers, separated by a thin oxide layer as shown in Fig. 3 [19]. Among two FM layers, the magnetic orientation of one layer is fixed and it is named as reference layer.

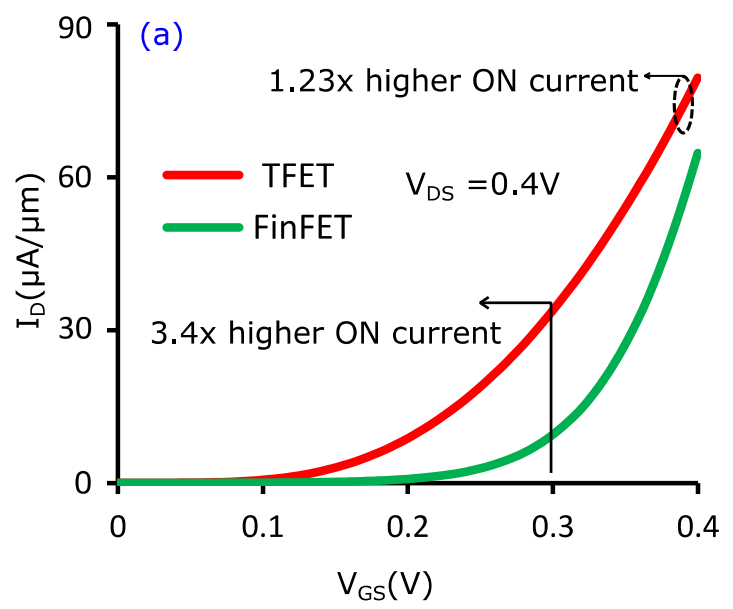

Table 1 Device parameters of InAs TFET and FinFET [30, 39]

\begin{tabular}{c|c}
\hline TFET Parameters & Value \\
\hline Channel length & $20 \mathrm{~nm}$ \\
\hline Effective oxide thickness (EoT) & $0.7 \mathrm{~nm}$ \\
\hline Channel thickness $\left(\mathrm{T}_{\mathrm{ch}}\right)$ & $5 \mathrm{~nm}$ \\
\hline Source Doping(InAs) & $4 \times 10^{19} \mathrm{~cm}^{-3}$ \\
\hline Drain Doping(InAs) & $6 \times 10^{17} \mathrm{~cm}^{-3}$ \\
\hline FinFET Parameters & Value \\
\hline Channel length & $20 \mathrm{~nm}$ \\
\hline Effective oxide thickness (EoT) & $0.7 \mathrm{~nm}$ \\
\hline Fin width $\left(W_{\text {Fin }}\right)$ & $8 \mathrm{~nm}$ \\
\hline Fin height $\left(\mathrm{H}_{\text {Fin }}\right)$ & $25 \mathrm{~nm}$ \\
\hline Source/Drain Doping & $1 \times 10^{20} \mathrm{~cm}^{-3}$ \\
\hline
\end{tabular}

Conversely, the magnetic orientation of other layer varies in same or opposite direction to that of the reference layer, named as free layer. Upon providing sufficient current through MTJ, the magnetic orientation of free layer can be changed. When the magnetic orientation of free layer and reference layer point in the same direction, MTJ exhibits lower resistance $\left(R_{P}\right)$ and it is called as parallel state. In contrast, if the magnetic orientation of free layer and reference layer point in opposite direction, MTJ exhibits higher resistance $\left(R_{A P}\right)$ and it is called as anti-parallel state. Due to the non-magnetic layer, MTJ exhibits variation in resistance, it is defined as tunnel magneto resistance (TMR) (Equation 1). MTJ is supposed to exhibit high TMR for faithful reproduction of stored value.

$$
T M R=\frac{R_{A P}-R_{P}}{R_{P}}
$$

Among several switching mechanisms available, spin transfer torque (STT) switching exhibits high TMR that is suitable to commercialize MTJs [20]. The perpendicular STT-MTJ devices eliminate the several challenges exhibited by in-plane devices. As a result, perpendicular STT-MTJ devices have attracted wide attention. This work explore a compact VerilogA model of $\mathrm{CoFeB} / \mathrm{MgO}$ perpendicular magnetic anisotropy STT-MTJ [21, 40]. The STT-MTJ described in this model is

(b)

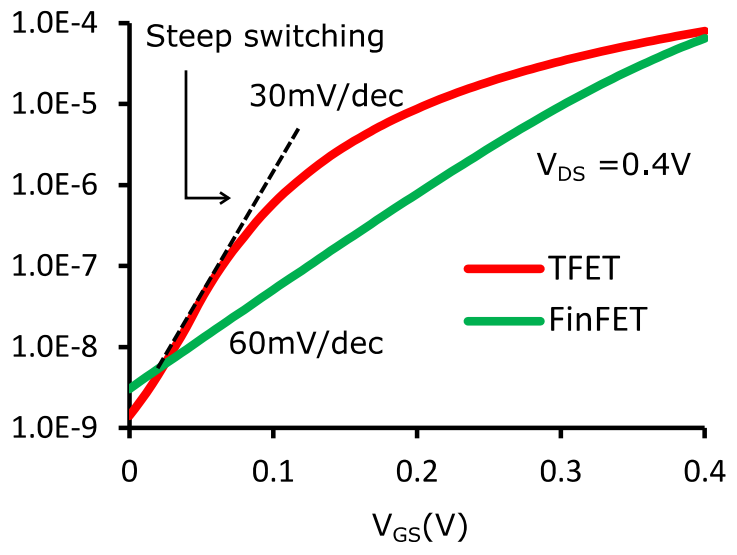

Fig. 2 The N-TFET $I_{D}-V_{G S}$ characteristics demonstrating (a) Higher ON current (b) Lower subthreshold swing (steep switching characteristics). 

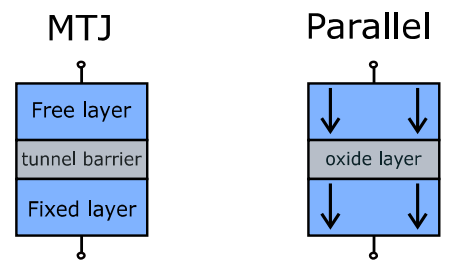

logic ' 1 '
Anti-parallel

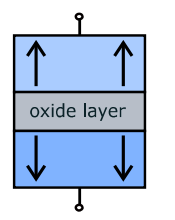

logic '0'

Fig. 3 MTJ device architecture with parallel and antiparallel states.

designed using two FM layers made of CoFeB and thin oxide layer made of $\mathrm{MgO}$. This model is compatible to industry standard Cadence environment and the parameters used are listed in Table 2. Therefore, the STTMTJ model is plugged, the circuits have been designed and analysed.

STT-MTJ require relatively large write current to change the state (high to low or low to high) that requires to maintain higher supply voltages. At scaled channel lengths

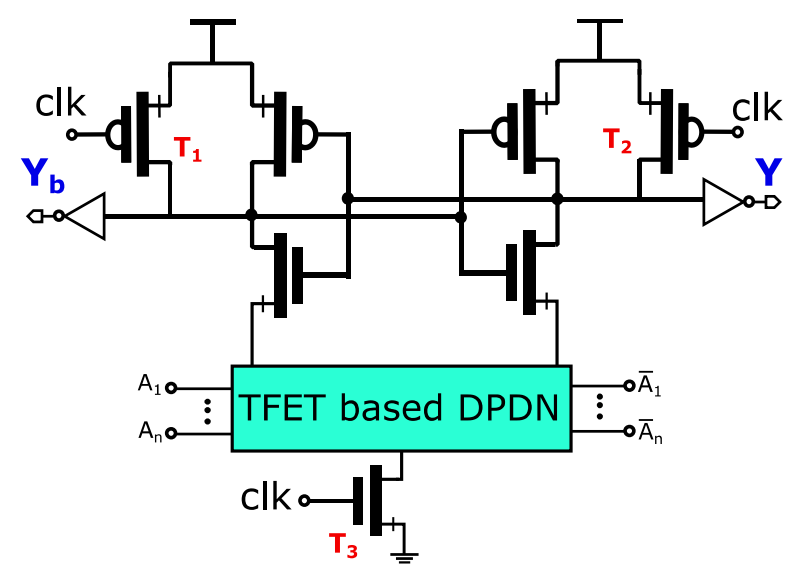

Fig. 4 Proposed TFET based SABL architecture with TFET DPDN

Table 2 Device parameters of perpendicular magnetic anisotropy STTMTJ [40]

\begin{tabular}{c|c}
\hline Parameters & Value \\
\hline Length of surface long axis & $20 \mathrm{~nm}$ \\
\hline Width of surface short axis & $20 \mathrm{~nm}$ \\
\hline TMR $(0)$ with Zero Volt Bias Voltage & $200 \%$ \\
\hline Thickness of free layer $\left(t_{s l}\right)$ & $1.3 \mathrm{~nm}$ \\
\hline Thickness of oxide barrier $\left(t_{o x}\right)$ & $0.85 \mathrm{~nm}$ \\
\hline
\end{tabular}

(scaled supply voltages), MOS devices exhibits lower ON current. As a result, the STT-MTJ exhibits higher propagation delay to change the state or the state cannot be changed. Thus, STT-MTJ based LiM cells exhibits higher energy consumption [25]. To reduce the energy consumption, this work explore TFET device (with higher ON current and lower subthreshold swing) in STT-MTJ based LiM circuits. Further, TFET and STT-MTJ based LiM circuits have been explored for logic encryption/locking technique.

\section{Energy Efficient and Secure TFET SABL based Crypto circuits}

This section demonstrates TFET based SABL gates design and analyses. Additionally, PRIDE S-box is designed and evaluated by performing DPA attack on it. Furthermore, the energy consumption of TFET based S-box design is benchmarked with FinFET based designs.

\subsection{TFET based SABL logic}

The proposed TFET SABL architecture that explores TFET based differential pull-down network (DPDN) is as shown in Fig. 4. The logic gates based on this architecture works in precharge and evaluation phase. In precharge phase $(\mathrm{clk}=0)$, transistors $T_{1}, T_{2}$ switch $\mathrm{ON}$ and $\mathrm{T}_{3}$ switches OFF. Consequently, the outputs $\left(\mathrm{Y}, \mathrm{Y}_{\mathrm{b}}\right)$ discharges to ground because of inverters. In the evaluation phase $(\mathrm{clk}=1)$, transistor
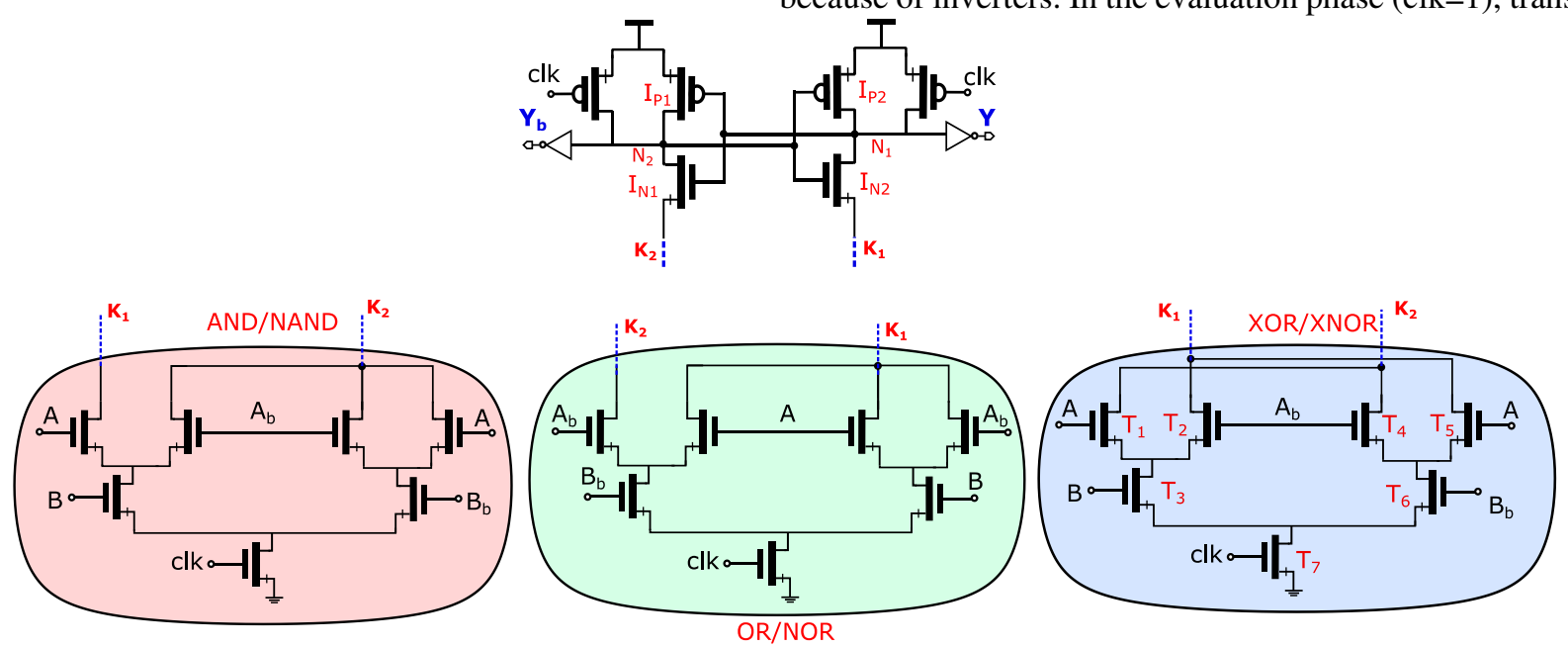

Fig. 5 TFET SABL based AND/NAND, OR/NOR and XOR/NOR designs corresponding to their TFET DPDN. 


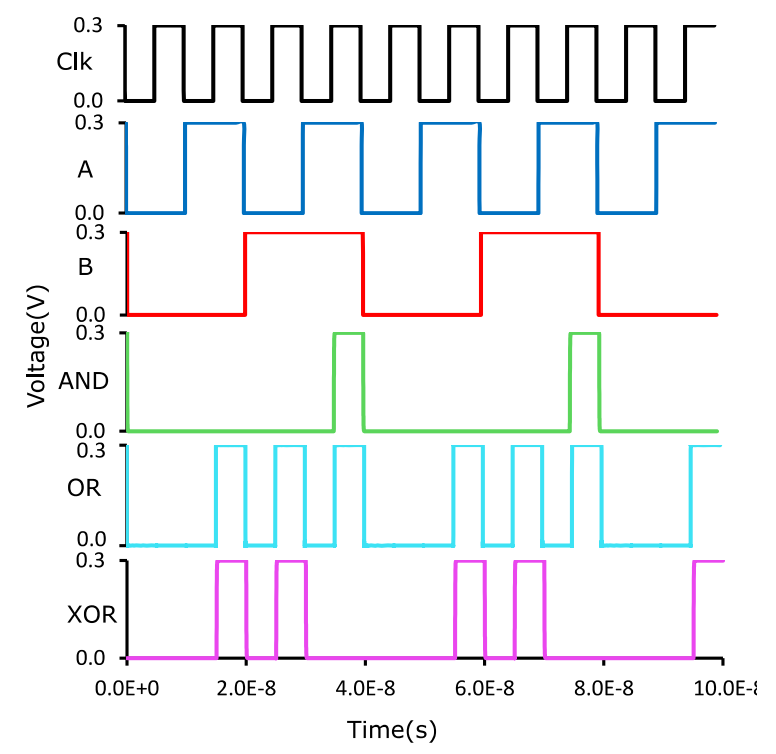

Fig. 6 Transient characteristics of TFET SABL gates at a supply voltage of $0.3 \mathrm{~V}$.

$\mathrm{T}_{3}$ switches ON and connects the TFET DPDN to ground. Depending upon the inputs applied to TFET based DPDN, the outputs $\left(\mathrm{Y}, \mathrm{Y}_{\mathrm{b}}\right)$ of $\mathrm{SABL}$ gates are evaluated. Exploring the SABL architecture, TFET AND/NAND, OR/NOR and XOR/NOR gates have been designed. The logic gate designs corresponding to their TFET DPDN circuits are shown in Fig. 5 where $K_{1}$ and $K_{2}$ are denoted to indicate the connection between pull-up and pull-down networks. For example, consider the TFET XOR gate wherein input $B$ is at logic ' 1 ' and $B_{b}$ is at logic ' 0 '. As input $A$ is at logic ' 0 ' and $A_{b}$ is at logic ' 1 ', the node $N_{1}$ discharges (through transistor $\mathrm{T}_{2}$ and $\mathrm{T}_{3}$ ) faster compared to node $\mathrm{N}_{2}$. As a result, the transistor $\mathrm{I}_{\mathrm{P} 1}$ in the inverter design (in pull-up network) switches $\mathrm{ON}$ and output $\mathrm{Y}_{\mathrm{b}}$ discharges to ground. When $Y_{b}$ discharges to gnd, $Y$ charges to supply voltage $\left(\mathrm{V}_{\mathrm{DD}}\right)$. Figure 6 shows the transient characteristics of TFET SABL gates at a supply voltage of $0.3 \mathrm{~V}$. It can be observed that TFET SABL gates exhibit proper functionality at ultra-low supply voltage of $0.3 \mathrm{~V}$ with $100 \mathrm{MHz}$ frequency. Table 3 presents the performance comparision of TFET SABL logic gates with FinFET SABL gates at a supply voltage of $0.3 \mathrm{~V}$. It is revealed that due to high $\mathrm{I}_{\mathrm{ON}} / \mathrm{I}_{\mathrm{OFF}}$ ratio, TFET SABL gates have been exhibited lower propagation delay compared to

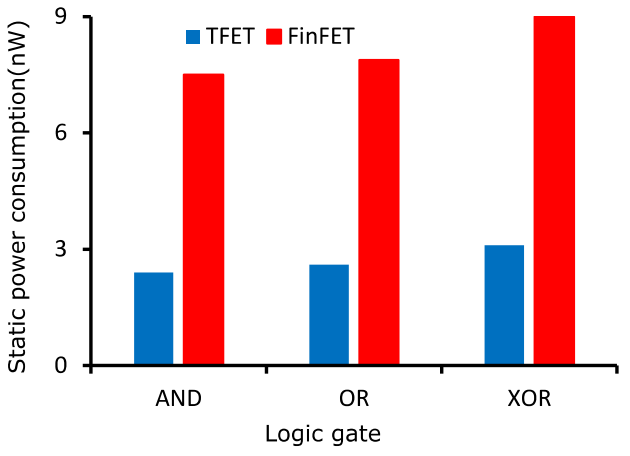

Fig. 7 Static power consumption comparision of TFET SABL gates with FinFET designs.

FinFET SABL logic gates. TFET SABL gates have also shown relatively lower power consumption and achieved $3 \times$ lower energy consumption compared to FinFET gates. Apart from this, the static power consumption of TFET SABL gates is calculated to test the vulnerability of SABL gates towards the static power side channel attacks. From Fig. 7, it can be observed that TFET SABL gates achieve approximately $3 \times$ lower static power consumption compared to FinFET based SABL at a supply voltage of $0.3 \mathrm{~V}$. Due to the lower static power consumption, TFET SABL gates can be robust against static power side channel attacks. From this, it can be concluded that TFET SABL gates exhibited better performance compared to FinFET designs at lower supply voltages.

\subsection{TFET SABL based PRIDE S-box design}

This section performs the DPA attack on the TFET SABL based PRIDE S-box. Further, the performance of TFET SABL Pride S-box is benchmarked with FinFET design.

\section{i. DPA mechanism on 4-bit PRIDE S-box}

PRIDE is a block cipher based on substitution and permutation networks with a 64-bit input/output and 128-bit key for encryption [18]. The complete encryption of PRIDE cipher is performed in 20 rounds. One round consists of mainly three operations including XOR with round key, 4-bit parallel substitution box (S-box) operations followed by permutations and linear operations. As it is widely accepted that S-box is a most vulnerable part of block cipher. Henceforth, this work considers 4-bit PRIDE S-box to perform differential power

Table 3 Performance comparision of TFET SABL gates with FinFET SABL gates at a supply voltage of $0.3 \mathrm{~V}$

\begin{tabular}{c|c|c|c|c|c|c}
\hline \multirow{3}{*}{ Gate } & \multicolumn{3}{|c|}{ TFET } & \multicolumn{3}{c}{ FinFET } \\
\cline { 2 - 7 } & $\begin{array}{c}\text { Power } \\
\text { consumption } \\
(\mu \mathrm{W})\end{array}$ & $\begin{array}{c}\text { Propagation } \\
\text { delay } \\
(\mathrm{ns})\end{array}$ & $\begin{array}{c}\text { Energy } \\
\text { consumption } \\
(\mathrm{fJ})\end{array}$ & $\begin{array}{c}\text { Power } \\
\text { consumption } \\
(\mu \mathrm{W})\end{array}$ & $\begin{array}{c}\text { Propagation } \\
\text { delay } \\
(\mathrm{ns})\end{array}$ & $\begin{array}{c}\text { Energy } \\
\text { consumption } \\
(\mathrm{fJ})\end{array}$ \\
\hline XOR & 1.1 & 0.5 & 0.55 & 1.13 & 2.1 & 2.373 \\
\hline AND & 0.812 & 0.6 & 0.49 & 0.83 & 1.94 & 1.61 \\
\hline OR & 0.95 & 0.52 & 0.49 & 0.92 & 1.9 & 1.75 \\
\hline
\end{tabular}




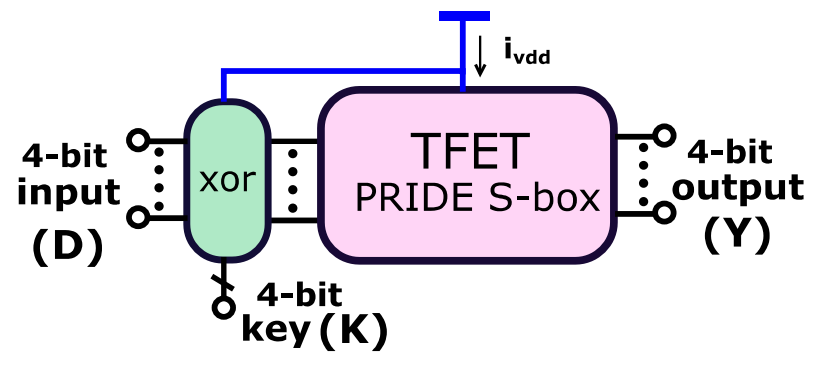

Fig. 8 Proposed TFET based PRIDE S-box design

analysis attack [18]. The 4-input and 4-output PRIDE Sbox is a combinational block and the relation between inputs and outputs is expressed as the boolean equations (2-5).

$$
\begin{aligned}
& y_{3}=x_{1} \oplus x_{3} \cdot x_{2} \\
& y_{2}=x_{0} \oplus x_{2} \cdot x_{1} \\
& y_{1}=x_{3} \oplus y_{3} \cdot y_{2} \\
& y_{0}=x_{2} \oplus y_{1} \cdot y_{2}
\end{aligned}
$$

The DPA attack is performed to retrieve the secret key information of crypto engine. The adversary contains the cryptographic algorithm with required input patterns to be applied. The DPA attack is performed on PRIDE S-box design which is shown in Fig. 8. Here XOR operations (as simple encryption) have performed between randomly generated inputs (D) and a 4-bit key (K). This produces the output that is applied to 4-bit S-box. Finally, S-box produces output $(\mathrm{Y})$ by performing substitution operation. The detailed DPA attack mechanism is explained as follows.

- Firstly, the S-box is designed using Cadence Virtuoso environment and the current traces ( $\mathrm{i}_{\mathrm{Vdd}}$ ) are recorded by applying random inputs (D) with a fixed key $\left(\mathrm{K}_{\mathrm{i}}\right)$.

- The recorded current traces are sampled with a rate of $\mathrm{T}$ ( $\mathrm{T}$ samples per trace) and arranged in a matrix $\mathrm{S}$ of size $\mathrm{D} \times \mathrm{T}$.

- In the next step, S-box algorithm is implemented using python 3 and the output values are calculated using inputs D (similar inputs as in first step) and all possible keys K.

- The obtained output values are mapped to hypothetical power consumption values using hamming distance (HD) model and represented by a matrix $\mathrm{H}$ which is of size $\mathrm{D} \times \mathrm{K}$. HD of the two successive output values is calculated by using the equation (6).

$$
\mathrm{HD}=\text { Hamming weight }\left(\mathrm{Y}_{i-1} \oplus \mathrm{Y}_{i}\right)
$$

- Finally, each column of $\mathrm{H}$ matrix is correlated with all the columns of current trace matrix $\mathrm{S}$. This results the correlation matrix (C) of size $\mathrm{K} \times \mathrm{D}$. The correct key used for encryption will show the highest value of correlation compared to other keys.

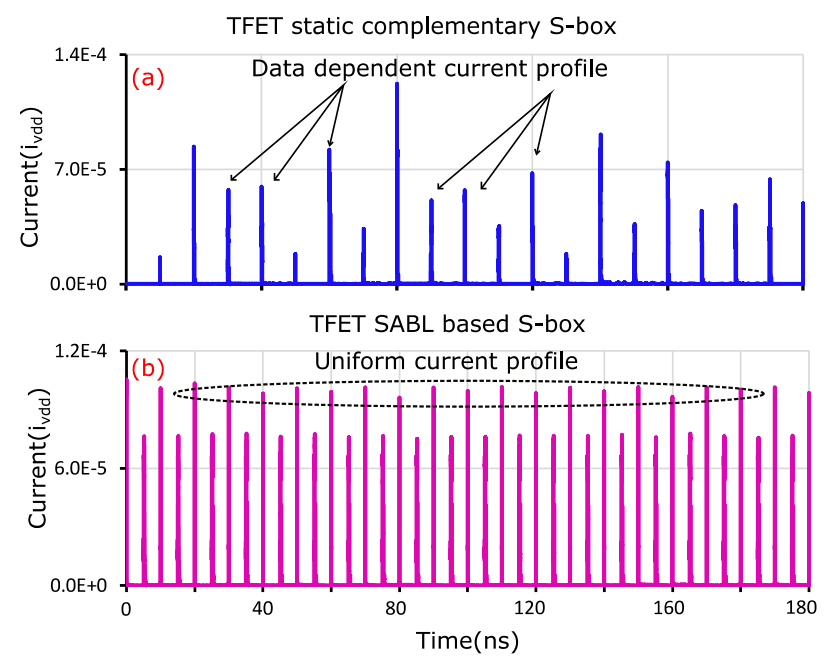

Fig. 9 Power traces of (a) TFET based static complementary S-box design (b) TFET SABL based S-box design.
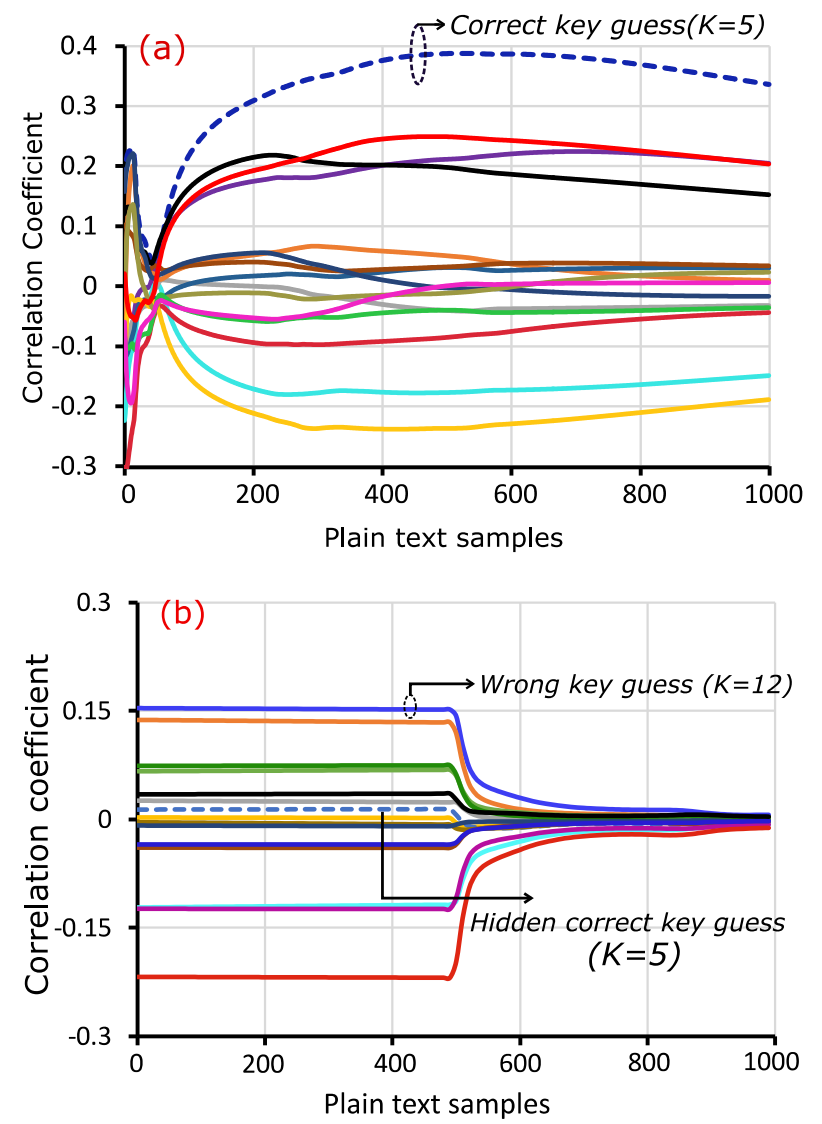

Fig. 10 Correlation coefficient of (a) TFET based static complementary S-box design (b) TFET SABL based S-box design 


\section{ii. Security evaluation of TFET SABL based PRIDE S-box}

The PRIDE S-box circuit is designed using proposed TFET SABL gates. The proposed TFET SABL based PRIDE S-box is compared with TFET based static complementary PRIDE S-box to highlight the security benefits. Both the designs have been implemented using $20 \mathrm{~nm}$ InAs TFET technology. Each design individually simulated using 100 random inputs with a fixed key $(\mathrm{K}=5)$. The power traces which are obtained from the TFET static and SABL based S-box circuits have been depicted in Fig. 9. From this, it can be observed that the power traces of TFET SABL based S-box circuit have proved to be uniform compared to TFET based static complementary design. The obtained power traces have been sampled with a rate of 1000 and arranged as a matrix $\mathrm{S}$. The resulted power trace matrix $\mathrm{S}$ is correlated with the hypothetical power values matrix $(\mathrm{H})$ by following the DPA mechanism. Figure 10(a) shows resultant correlation coefficients of TFET based static complementary S-box design with all possible keys. It can be seen that the DPA attack performed on this design is successful and shows highest correlation coefficient for the correct key $(\mathrm{K}=5)$. Besides, the DPA attack performed on TFET SABL based S-box design is observed to be unsuccessful as shown in Fig. 10(b). The correlation coefficient of wrong key $(\mathrm{K}=12)$ is observed to be high compared to the original key $(\mathrm{K}=5)$. Moreover, the correlation coefficient of original key is hidden in the analysis result and cannot be observed by the adversary. This robustness is obtained from the favourable SABL structure and the sharp switching behaviour of TFET device.

\section{iii. Performance benchmarking}

The performance of TFET SABL based S-box design is benchmarked with equivalent FinFET SABL based S-box design as shown in Table 4. At a supply voltage of $0.3 \mathrm{~V}$, TFET and FinFET based designs have shown an energy consumption of 13.09fJ and 41.229f respectively. Thus, TFET SABL based S-box design exhibited 3.15x lower energy consumption compared to FinFET design at a supply voltage of $0.3 \mathrm{~V}$. Moreover, static power

Table 4 Performance comparison of TFET SABL based S-box design with equivalent FinFET SABL based S-box design at a supply voltage of $0.3 \mathrm{~V}$.

\begin{tabular}{c|c|c}
\hline Parameter & $\begin{array}{c}\text { TFET SABL } \\
\text { based S-box }\end{array}$ & $\begin{array}{c}\text { FinFET SABL } \\
\text { based S-box }\end{array}$ \\
\hline $\begin{array}{c}\text { Power } \\
\text { consumption( } \boldsymbol{\mu} \mathbf{W})\end{array}$ & 6.3 & 6.7 \\
\hline $\begin{array}{c}\text { Static power } \\
\text { consumption(nW) }\end{array}$ & 17.53 & 61.35 \\
\hline $\begin{array}{c}\text { Propagation } \\
\text { delay(ns) }\end{array}$ & 2.12 & 6.17 \\
\hline $\begin{array}{c}\text { Energy } \\
\text { consumption(fJ) }\end{array}$ & 13.09 & 41.339 \\
\hline
\end{tabular}

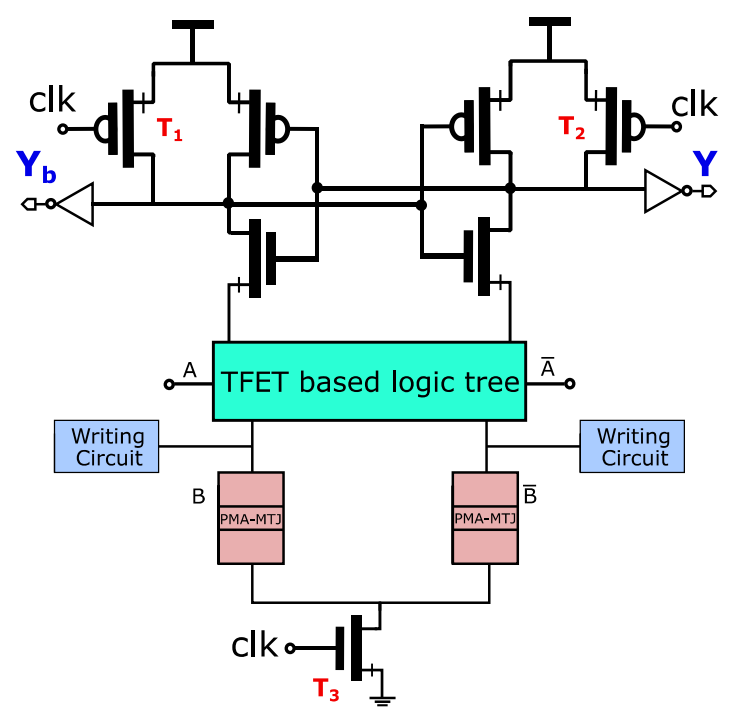

Fig. 11 Proposed architecture of STT-MTJ and TFET LiM cells

consumption of TFET SABL based S-box design is observed to be $3.5 \times$ lower compared to FinFET design. Due to this, TFET SABL based crypto circuits can exhibit lower vulnerability to static power side channel attacks.

\section{STT-MTJ and TFET Logic-in-Memory (LiM) cells for Logic locking}

This section presents the STT-MTJ and TFET based LiM gates and benchmarked with the STT-MTJ and FinFET based designs. Further, these gates are applied to logic locking/encryption and energy consumption is calculated. Moreover, the fabrication compatibility and challenges of TFET and STT-MTJ have been discussed.

\subsection{STT-MTJ and TFET LiM based logic gates}

The proposed STT-MTJ and TFET LiM cells are identical to SABL gates wherein STT-MTJ devices are explored to store one-bit input as shown in Fig. 11. This stored input can be accessed by logic tree and the final output of logic-in-memory cell is produced with the help of pre-charge sense amplifier. An additional writing circuit is used to write the data into STTMTJ devices. The STT-MTJ and TFET LiM cell works in two phases that is similar to TFET SABL gates. When the clock signal is at logic ' 0 ' (clk=0), the sense amplifier works in precharge phase. In this phase, logic tree is disconnected from the sense amplifier, the transistors $T_{1}$ and $T_{2}$ switches ON. As a result, outputs $\mathrm{Y}$ and $\mathrm{Y}_{\mathrm{b}}$ of the cell discharge to logic 0 . When the clock signal is at logic ' 1 ' (clk=1), the sense amplifier works in evaluation phase. In this phase, with the resistance difference between the MTJs and depending upon input, the charging/discharging speed of the two braches varies. As a result, the sense amplifier evaluates one output to be logic ' 1 ' while the other output to be at logic ' 0 '.

Figure 12 shows the STT-MTJ and TFET based AND/NAND, OR/NOR, and XOR/XNOR LiM gates corresponding to 

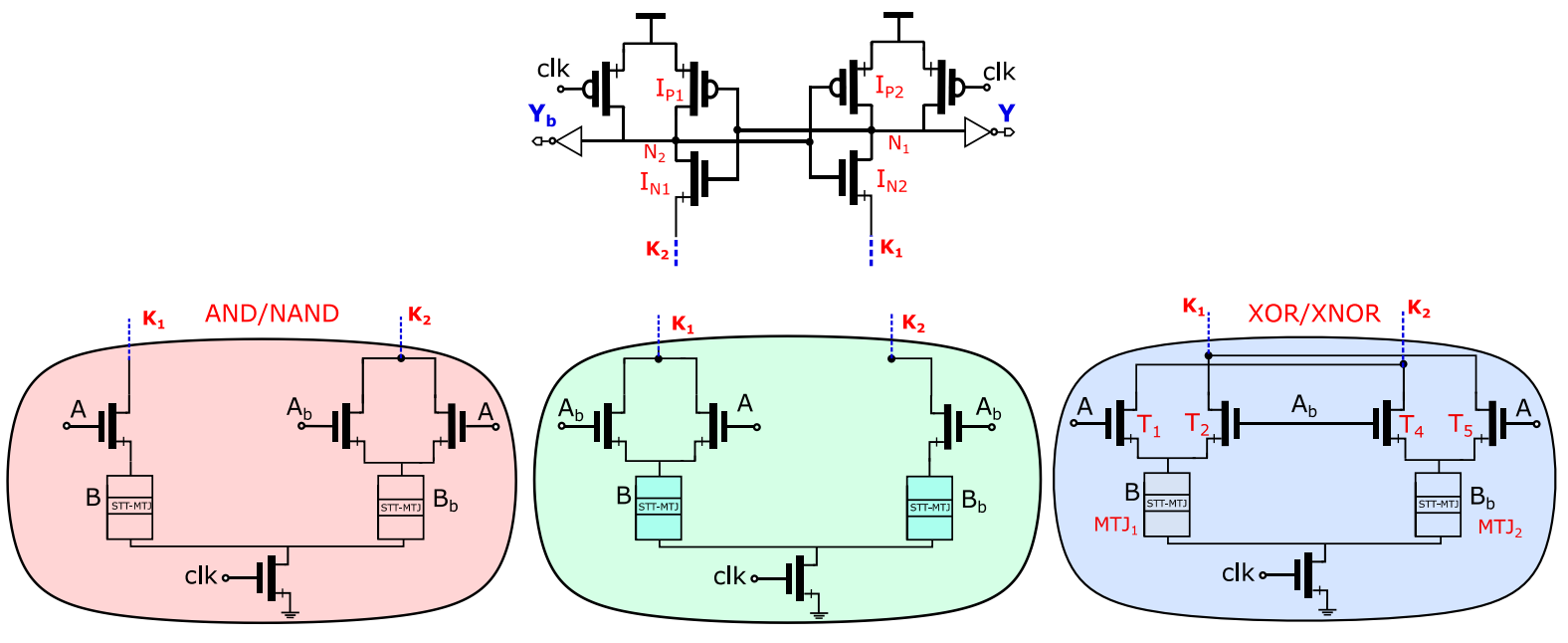

OR/NOR

Fig. 12 STT-MTJ and TFET based AND/NAND, OR/NOR, and XOR/XNOR LiM gates.

different logic tree structures where $\mathrm{K}_{1}$ and $\mathrm{K}_{2}$ are denoted to show the connection between pull-up and pull-down networks. More specifically, consider XOR gate in which two MTJ devices are used to store the input ' $\mathrm{B}$ ' and its compliment. For example, consider that $\mathrm{MTJ}_{1}$ (B is at logic ' 1 ') and $\mathrm{MTJ}_{2}\left(\mathrm{~B}_{\mathrm{b}}\right.$ is at logic ' 0 ') are in parallel and anti-parallel configurations respectively. Consequently, resistance of $\mathrm{MTJ}_{1}$ is lesser than that of $\mathrm{MTJ}_{2}$. With relatively lower resistance, current through $\mathrm{MTJ}_{1}$ is greater than the $\mathrm{MTJ}_{2}$. As input A is at logic ' 1 ' and $\mathrm{A}_{\mathrm{b}}$ is at logic ' 0 ', the charge stored at node $\mathrm{N}_{2}$ discharge (through transistor $\mathrm{T}_{1}$ ) faster compared to node $\mathrm{N}_{1}$. As a result, the transistor $\mathrm{I}_{\mathrm{P} 2}$ in the inverter design (pull-up network) switches $\mathrm{ON}$ and output node $\mathrm{N}_{1}$ charges to $\mathrm{V}_{\mathrm{DD}}$. Due to this, $\mathrm{Y}$ and $\mathrm{Y}_{\mathrm{b}}$ result into to gnd and $\mathrm{V}_{\mathrm{DD}}$ respectively.

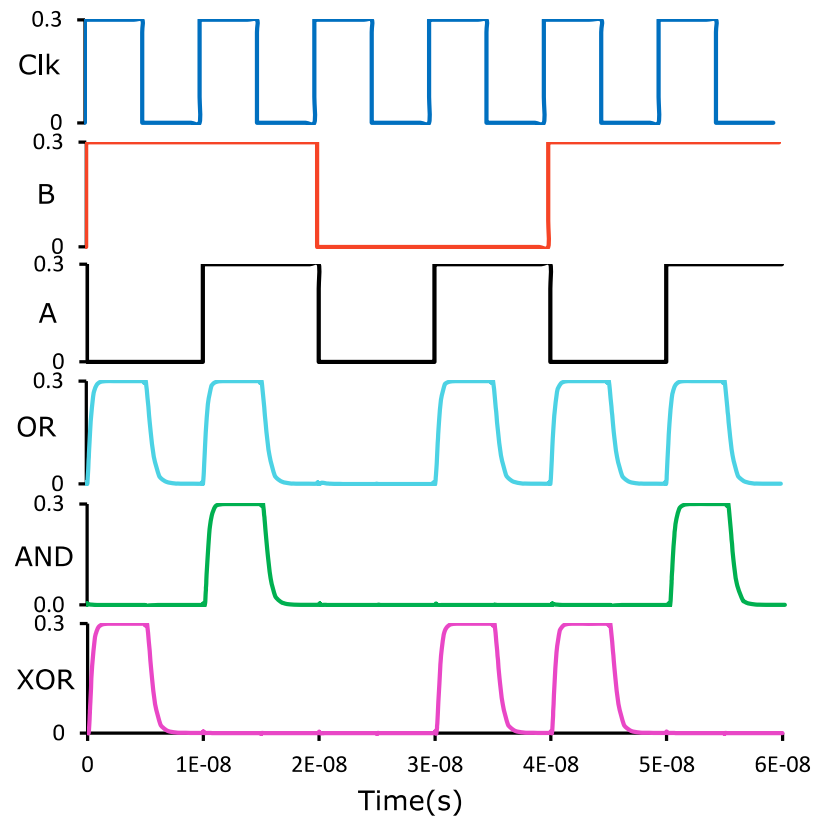

Fig. 13 Transient characteristics of proposed STT-MTJ and TFET LiM cells based logic gates at a supply voltage of $0.3 \mathrm{~V}$.
Figure 13 shows the transient characteristics of logic gates at a supply voltage of $0.3 \mathrm{~V}$. From this, it can be observed that STTMTJ and TFET based logic gates exhibited proper functionality at low supply voltage. Figure 14 shows the energy consumption comparison of STT-MTJ and TFET based logic gates with equivalent STT-MTJ and FinFET designs at a supply voltage $0.3 \mathrm{~V}$. STT-MTJ and TFET based gates achieved $4 \times$ lower energy consumption compared to STT-MTJ and FinFET designs. With the higher ON current of TFET devices, STT-MTJ exhibited lower propagation delay in changing its state. As a result, proposed LiM based gates achieve lower energy consumption. Further, these gates are explored for energy efficient logic encryption/locking application.

\subsection{STT-MTJ and TFET LiM cells based logic locking}

Logic locking is a hardware security technique that protects the circuit/design by adding key based gates into original design.

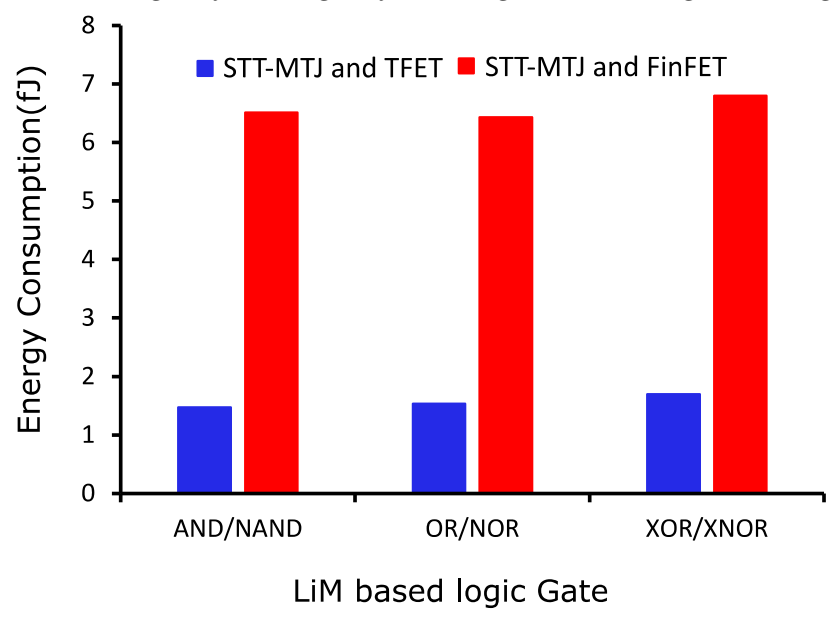

Fig. 14 Energy consumption comparison of STT-MTJ and TFET based logic gates with equivalent STT-MTJ and FinFET designs at a supply voltage $0.3 \mathrm{~V}$. 
(a) Conventional logic encryption Original circuit/system

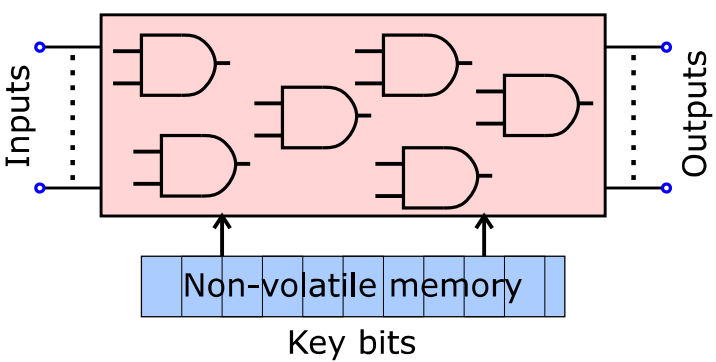

(b) LiM gates based logic encryption Original circuit/system

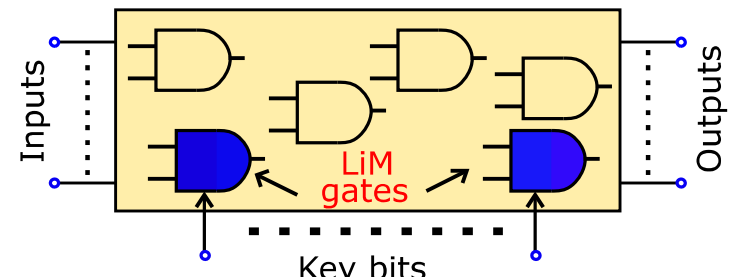

Key bits

Fig. 15 Block diagram of (a) Conventional logic locking (b) Proposed LiM gates based logic locking

STT-MTJ/TFET based NAND gate

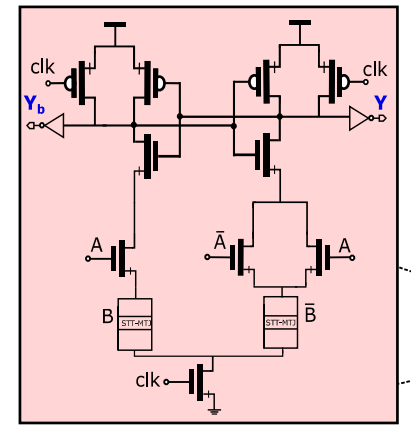

ISCAS C17 Benchmark

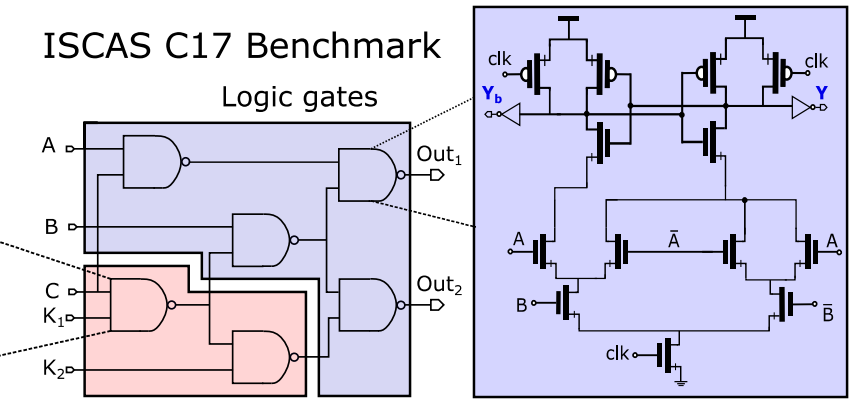

LiM based gates
TFET SABL based NAND gate

Fig. 16 STT-MTJ and TFET based ISCAS C17 benchmark explored for logic locking application.

Consequently, the circuit/system cannot provide proper functionality without the correct key input. Figure 15 shows the block diagram of conventional and proposed LiM based logic locking in which both input and key will be provided to the design and proper functionality can be achieved upon applying correct key. In conventional logic locking technique a separate non-volatile memory is used to store the key bits. Recent research revealed that fetching of the data from external memory exhibits large energy overhead [19-20]. To reduce the energy overhead, this paper explore STT-MTJ and TFET LiM cells based logic locking wherein the STT-MTJ and TFET LiM cells are used to store and process the data. For example, consider ISCAS C17 benchmark (Fig. 16) that is explored for logic locking application [41]. This design uses six two inputNAND gates wherein two NAND gates are designed using STT-MTJ and TFET LiM cells that are operated by key inputs $\left(K_{I}\right.$ and $\left.K_{2}\right)$ as shown in Fig. 16. As a result, the STT-MTJ stores key bits and the logic is processed using TFET based logic tree. The remaining four NAND gates are designed using TFET SABL based NAND gates as shown in Fig. 16. The $\mathrm{Out}_{1}$ and $\mathrm{Out}_{2}$ of ISCAS C17 circuit are expressed as equations 7 and 8 .

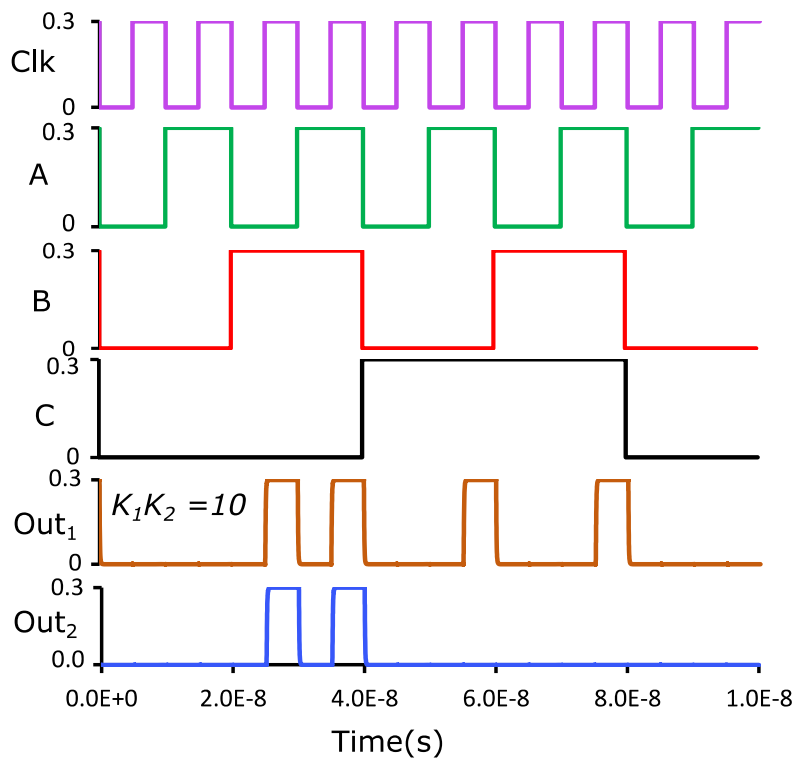

Fig. 17 Transient characteristics of STT-MTJ and TFET based logic encryption circuit with key bits $K_{l} K_{2}=10$. 


$$
\begin{aligned}
& \text { Out }_{1}=A \cdot C+B \cdot\left(\overline{C \cdot K_{1}}\right) \\
& \text { Out }_{2}=\left(\overline{C \cdot K_{1}}\right) \cdot\left(B+K_{2}\right)
\end{aligned}
$$

For example, the key bits $K_{l} K_{2}$ are fixed as " 10 ", the equations 7 and 8 are simplified as equations 9 and 10

$$
\begin{aligned}
& O u t_{1}=A \cdot C+B \cdot \bar{C} \\
& O u t_{2}=B \cdot \bar{C}
\end{aligned}
$$

Figure 17 shows the transient characteristics of proposed ISCAS C17 based circuit at a supply voltage of $0.3 \mathrm{~V}$. With key bits of $K_{1} K_{2}=$ " 10 ", the circuit shows correct functionality as mentioned in equation 9 and 10 . The energy consumption of STT-MTJ and TFET LiM cells based C17 circuit is calculated at a supply voltage of $0.3 \mathrm{~V}$. It can be observed that STT-MTJ and TFET based logic encryption circuit achieves a lower energy consumption of 6.96fJ. On the other hand, STT-MTJ and FinFET based logic encryption circuit shows energy consumption of $21.64 \mathrm{fJ}$. From this, it can be seen that proposed design shows $3.11 \times$ lower energy consumption compared to FinFET designs. STT-MTJ and TFET LiM based logic encryption achieves lower energy consumption due to the higher ON current and low leakage characteristics of TFET at lower supply voltages.

\subsection{Fabrication Compatibility of STT-MTJ with TFET}

The homo-junction TFET and CMOS FinFET devices have been fabricated using identical manufacturing process since architecture of both devices is observed to be similar [28]. It is experimentally proved that complementary TFET devices fabricated in standard CMOS foundry exhibited higher compatibility for commercial production and enhanced flexibility for heterogeneous TFET-CMOS systems [28]. Several research groups have demonstrated the benefits of mixed MOSFET-TFET circuits/systems and corresponding layout rules with both simulations and experimental fabrication [31]. Besides, STT-MTJ exhibits higher compatibility with CMOS process by fabricating them in back-end-of-the-line. This encouraged the researches to explore logic-in-memory architectures that achieve low area and energy overheads. Recent research demonstrated the heterogeneous 3D integration for STT-MTJ based memory-on-logic applications that separates memory, logic blocks and stacks memory tire on top of logic block [20]. This STT-MTJ based 3D integration achieved silicon area saving with performance gain.

CMOS exhibited higher compatibility with STT-MTJ and TFET is fabricated using identical manufacturing like CMOS. Therefore, the TFET technology can show ease of integration and flexibility in manufacturing with STT-
MTJ. However, secondary effects including ambipolarity, $\mathrm{p}-\mathrm{i}-$ $\mathrm{n}$ forward current and enhanced miller effect still exist in TFET which make it unpopular and less suitable for commercialization now [31]. More research efforts are require to make these emerging technology integration a reality.

\section{Conclusion}

Existing CMOS SABL based circuits and CMOS based STTMTJ LiM circuits exhibited large energy consumption with increased vulnerability towards hardware security attacks. To reduce the energy consumption, emerging TFET is introduced into these circuits that achieved ultra-low energy consumption with enhanced security. TFET SABL based gates and PRIDE S-box design achieve approximately $3 \times$ lower energy consumption compared to FinFET designs while maintaining higher DPA resilience. Moreover, TFET SABL based crypto systems with lower static power consumption can show vulnerability to static power side-channel attacks. Additionally, proposed STT-MTJ and TFET LiM gates achieves $4 \times$ lower energy consumption compared to STT-MTJ and FinFET designs. The proposed STT-MTJ and TFET LiM gates have been explored in logic encryption/locking technique that shows $3 \times$ lower energy consumption compared to STTMTJ and FinFET designs. STT-MTJ and TFET based circuits have exhibited higher compatibility in fabrication. However, STT-MTJ and TFET devices exhibited several second order effects including low reliability, process variations and other leakages (ambipolarity and p-i-n forward current) that make commercialization of these devices difficult. More research efforts are required in future from the device-circuit community to resolve the existing challenges.

\section{References}

1. Li, A., Shinde, Y., Shi, J., Ye, X., Y. Li., and W. Z. Song.: System Statistics Learning-Based IoT Security: Feasibility and Suitability. IEEE J. Internet of Things. 6(4), 6396-6403 (2019).

2. The Cost of Malicious Cyber Activity to the US Economy, W. House, 2018. [Online].

Available:https://www.whitehouse.gov/wpcontent/uploads/2018/03/T he-Cost-of-Malicious-Cyber-Activity-to-the-U.S.-Economy.pdf

3. Bartock, M., Souppaya, M., Savino, R., Knoll, T., Shetty, U., Cherfaoui, M., Yeluri, R. and Scarfone, K.: Hardware-Enabled Security for Server Platforms: Enabling a Layered Approach to Platform Security for Cloud and Edge Computing Use Cases. National Institute of Standards and Technology. (pp. 39-39).

4. Roshanisefat, S., Kamali, H. M., Homayoun, H., and Sasan, A.: SAThard Cyclic Logic Obfuscation for Protecting the IP in the Manufacturing Supply Chain. IEEE Trans. VLSI Syst. 28(4), 954-967 (2020).

5. Prinetto, P., and Roascio, G.: Hardware Security, Vulnerabilities, and Attacks: A Comprehensive Taxonomy. In ITASEC.177-189 (2020).

6. De, P., Mandal, C., and Prampalli, U.: Path-Balanced Logic Design to Realize Block Ciphers Resistant to Power and Timing Attacks. IEEE Trans. VLSI Syst. 27(5), 1080-1092 (2019).

7. Wang, W., Standaert, Y. F. X., Liu, J., Guo, Z., and Gu, D.: RidgeBased DPA: Improvement of Differential Power Analysis For Nanoscale Chips. IEEE Trans. Inform. Forensics and Security. 13(5), 1301-1316 (2018).

8. Rossi, D., Tenentes,V., Yang, S., Khursheed S., and Al-Hashimi, B. 
M.: Aging Benefits in Nanometer CMOS Designs. In IEEE Trans. on Circuits and Systems II: Express Briefs. 64(3), 324328(2017).

9. De Cnudde, T., Nikova, S.: Securing the present block cipher against combined side-channel analysis and fault attacks. IEEE Trans. Very Large Scale Integ. Syst., 25(12), 3291-3301(2017).

10. Shanmugham, S. R., and Paramasivam, S.: Survey on power analysis attacks and its impact on intelligent sensor networks. IET Wireless Sensor Systems, 8(6), 295-304 (2018).

11. Tena-Sanchez, E., Castro, J., and Acosta, A. J.: A Methodology for Optimized Design of Secure Differential Logic Gates for DPA Resistant Circuits. IEEE J. Emerg. Select. Topics Circuits Syst. 4(2), pp. 203-215 (2014).

12. Kumar, S. D., Thapliyal, H., and Mohammad, A.: FinSAL: A novel FinFET based Secure Adiabatic Logic for energy-efficient and DPA resistant IoT devices. IEEE Trans. Comput.-Aided Design Integr. Circuits and Syst. 37(1), 2017.

13. Avital, M., Dagan, H., Keren, O., and Fish, A.: Randomized Multitopology Logic Against Differential Power Analysis. In IEEE Trans. on VLSI Syst., 23(4), 702-711(2015).

14. Tanimura, K., and Dutt, N. D.: HDRL: Homogeneous Dual-Rail Logic for DPA Attack Resistive Secure Circuit Design. In IEEE Embedded Systems Letters, 4(3), 57-60 (2012).

15. Kumar, S. D., Thapliyal, H., and Mohammad, A.: EE-SPFAL: A Novel Energy-Efficient Secure Positive Feedback Adiabatic Logic for DPA Resistant RFID and Smart Card. IEEE Trans. Emerging Topics in Comput. 7(2), 281-293(2019).

16. Saini, H., and Gupta, A.: Constant Power Consumption Design of Novel Differential Logic Gate for Immunity against Differential Power Analysis. IET Circuits, Devices \& Syst. 13(1), 103-109(2018).

17. Bellizia, D., Bongiovanni, S., Monsurro, P., Scotti, G., and Trifiletti, A.: Univariate Power Analysis Attacks Exploiting Static Dissipation of Nanometer CMOS VLSI Circuits for Cryptographic Applications. IEEE Trans. Emerging Topics in Comput. 5(3), 329-339(2017).

18. Delgado-Lozano, I. M., Tena-Sanchez, E., Nunez J., and Acosta, A. J.: Design and Analysis of Secure Emerging Crypto-Hardware using HyperFET Devices. IEEE Trans. Emerg. Topics in Comput. (2020).

19. Hanyu, T., Endoh, T., Suzuki, D., Koike, H., Ma, Y., Onizawa, N., Natsui, M., Ikeda, S. and Ohno, H.: Standby-power-free integrated circuits using MTJ-based VLSI computing. Proc. of the IEEE. 104(10), 1844-1863(2016).

20. Zhu, L., Bamberg, L., Agnesina, A., Catthoor, F., Milojevic, D., Komalan, M., Ryckaert, J., Garcia-Ortiz, A. and Lim, S.K.: Heterogeneous 3D Integration for a RISC-V System With STTMRAM. IEEE Computer Architecture Lett. 19(1), 51-54(2020).

21. Shi, Y., Oh, S., Huang, Z., Lu, X., Kang, S.H. and Kuzum, D.: Performance Prospects of Deeply Scaled Spin-transfer Torque Magnetic Random-access Memory for In-memory Computing. IEEE Electron Device Lett. (2020).

22. Deng, E., Zhang Y., Klein, J. O., Ravelsona, D., Chappert, C., Zhao, W.: Low power magnetic full-adder based on spin transfer torque MRAM. IEEE Trans. on Magnet. 49(9), 4982-7(2013).

23. Deng, E., Zhang, Y., Kang, W., Dieny, B., Klein, J. O., Prenat, G., Zhao, W.: Synchronous 8-bit non-volatile full-adder based on spin transfer torque magnetic tunnel junction. IEEE Transactions on Circuits and Systems I: Regular Papers. 62(7), 1757-65(2015).

24. Cai, H., Wang, Y., Naviner, L. A., Zhao, W.: Robust ultralow power non-volatile logic-in-memory circuits in FD-SOI technology. IEEE Trans. on Circuits and Systems I: Regular Papers. 64(4), 847-57(2016).

25. Natsui, M., Suzuki, D., Sakimura, N., Nebashi, R., Tsuji, Y., Morioka, A., Sugibayashi, T., Miura, S., Honjo, H., Kinoshita, K., Ikeda, S.: Nonvolatile logic-in-memory LSI using cycle-based power gating and its application to motion- vector prediction. IEEE Journal of Solid-State Circuits. 50(2), 47689(2014).

26. Kumar, S. D., and Thapliyal, H.: Exploration of Non-Volatile MTJ/CMOS Circuits for DPA-Resistant Embedded Hardware. IEEE Trans. Magnet., 55(12), 1-8(2019).

27. Resta, G.V., Leonhardt, A., Balaji, Y.: Devices and circuits using novel 2-D materials: a perspective for future VLSI systems. IEEE Trans. VLSI Syst. 27(7), 1486-1503(2019).

28. Gopireddy, B., Skarlatos, D., Zhu, W., and Torrellas, J.: HetCore: TFET-CMOS hetero-device architecture for CPUs and GPUs. ACM/IEEE 45th Annual International Symposium on Computer Architecture. 802-815(2018).

29. Kino, H., Fukushima, T., and Tanaka, T.: Generation of STDP With Non-Volatile Tunnel-FET Memory for Large-Scale and Low-Power Spiking Neural Networks. In IEEE Journal of the Electron Devices Society. 8, 1266-1271 (2020).

30. Aditya, J., Vallabhaneni, H., and Vaddi, R.: Reliability enhancement of a steep slope tunnel transistor based ring oscillator designs with circuit interaction. IET Circuits, Devices \& Syst. 10(6), 522527(2016)

31. Lin Z., et al.: Challenges and Solutions of the TFET Circuit Design, IEEE Trans. on Circuits and Systems I: Regular Papers. 67(12), 49184931(2020).

32. Aditya, J., et al.: Tunneling Field Effect Transistors for Enhancing Energy Efficiency and Hardware Security of IoT Platforms: Challenges and Opportunities. In Proc. IEEE Int. Symp. Circuits and Systems (ISCAS). 1-5(2018).

33. Bi, Y., et al.: Tunnel FET current mode logic for DPA-resilient circuit designs. IEEE Trans. Emerg. Top. Comput. 5(3), 340-352(2017).

34. Thapliyal, H., Varun, T. S. S., and Dinesh Kumar, S.: Low-Power and Secure Lightweight Cryptography Via TFET-Based Energy Recovery Circuits. In Proc. IEEE Int. Conf. Rebooting Comput., Washington. 14(2017).

35. Chen, A., et al:: Using emerging technologies for hardware security beyond PUFs. In IEEE Design, Automation \& Test Conf. \& Exhibition, Dresden. 1544-1549(2016).

36. Aditya, J., Majumder, M. K., Sahoo, S. K., and Vaddi, R. Tunnel FET Ambipolarity-Based Energy Efficient and Robust True Random Number Generator Against Reverse Engineering Attacks. IET Circuits, Devices \& Syst., 13(5), 689-695(2019).

37. Aditya, J., Majumder, M. K., Sahoo, S. K., and Vaddi, R. Low area overhead DPA countermeasure exploiting tunnel transistor-based random number generator. IET Circuits, Devices \& Syst., 14(5), 640647(2020).

38. Aditya, J., Majumder, M. K., Sahoo, S. K., and Vaddi, R.: Tunnel FET-based ultralow-power and hardware-secure circuit design considering $\mathrm{p}-\mathrm{i}-\mathrm{n}$ forward leakage. International Journal of Circuit Theory and Applications, 48(4), 524-538(2020).

39. Liu, H., Narayanan, V., Datta, S, et al.: III-V Tunnel FET model. version 1.0.1.2015 https://nanohub.org/publications/12/2

40. Zhang, Y., et al., "Compact Modeling of Perpendicular-Anisotropy $\mathrm{CoFeB} / \mathrm{MgO}$ Magnetic Tunnel Junctions," in IEEE Trans. on Electron Devices. 59(3), 819-826 (2012).

41. Hansen, M. C., Yalcin, H., and Hayes, J. P.: Unveiling the ISCAS-85 benchmarks: A case study in reverse engineering. IEEE Design \& Test of Comput., 16(3), 72-80, (1999). 
Journal of ComputationalElectronics 